\title{
Generation of coherent confined acoustic phonons by drifting electrons in quantum wire
}

\author{
A.A. Demidenko, V.A. Kochelap \\ Institute of Semiconductor Physics, NAS Ukraine, 45 Prospect Nauki, Kyiv, 03028, Ukraine \\ E-mail: kochelap@nikal.pp.kiev.ua
}

\begin{abstract}
We developed a quantum theory of generation of coherent confined acoustic phonons by one-dimensional electrons drifting in a quantum wire. A general formula for the factor of amplification of the phonon flux, $\alpha$, is derived. When the electron drift velocity, $V_{d}$, is greater than the sound phase velocity, $S$, then at low temperatures the above generation occurs only in a narrow range of phonon wave vectors, $q \sim 2 k_{\mathrm{F}}\left[1 \pm\left(V_{d}-S\right) / V_{F}\right]$, where $k_{F}\left(V_{F}\right)$ is the wave vector (Fermi velocity) of one-dimensional electrons. Estimation gives for $\alpha$ the value of about $10^{3} \mathrm{~cm}^{-1}$ in the subTHz frequency range.
\end{abstract}

Keywords: acoustic wave localization, acoustic wave amplification, electron drift, quantum wire. Paper received 18.02.00; revised manuscript received 13.11.00; accepted for publication 12.12.00.

1. In [1] we have constructed solutions to equations of the theory of elasticity in the form of acoustic waves localized in a quantum well. We considered their interaction via deformation potential with electrons drifting in a quantum well. This interaction results in amplification of the above acoustic waves. Similar problem has been solved in [2] for the case of piezoelectric interaction. In both [1] and [2] it was assumed that the acoustic wavelength is big as compared to the Fermi electron wavelength, and so the acoustic wave was treated using the quasi-classical approach.

In [3] we considered interaction via deformation potential between the flux of quantized localized phonons and two-dimensional (2D) electrons drifting in a quantum well. We have derived an analytical expression for the factor of amplification (generation) of coherent phonons. Here we give solution to a similar problem for the case of $1 \mathrm{D}$ electrons in a quantum wire.

2. Let us consider the following composite medium. A cylinder (whose axis is oriented along $z$ ) of radius $R$ is made of material $A$ with the following parameters: density $\rho$, Lamé constants $\lambda$ and $\bar{\mu}$. This cylinder is placed in a medium made of material $A$ whose corresponding parameters are $\bar{\rho}, \bar{\lambda}$ and $\bar{\mu}$.

We shall seek for solutions to equations of the theory of elasticity for the above composite medium. These solutions are of the form of acoustic waves that are localized near the cylinder and die off as $\rho>R$ grows. The volume equations of motion are as follows $[4,5]$ : $\rho \ddot{\vec{U}}=\mu \Delta \vec{U}+(\lambda+\mu) \nabla(\nabla \cdot \vec{U})$

here $\vec{U}$ is the vector of medium elastic displacement. Following $[6,7]$, let us express $\vec{U}$ through potentials:

$\vec{U}=\nabla \phi_{0}+\operatorname{rot} \phi_{1} \vec{e}_{z}+\operatorname{rotrot} \phi_{2} \vec{e}_{z} ;$

here $\vec{e}_{z}$ is the ort along $z$.

To satisfy the Eq. (1), the potentials $\phi_{j}(j=0,1,2)$ are to obey the following equations:

$\rho \ddot{\phi}_{0}=(\lambda+2 \mu) \Delta \phi_{0} ; \rho \ddot{\phi}_{1,2}=\mu \Delta \phi_{1,2}$

or, assuming their temporal dependence to be $\propto e^{-i \omega t}$,

$\Delta \phi_{0}=-q_{L}^{2} \phi_{0} ; \quad \Delta \phi_{1,2}=-q_{T}^{2} \phi_{1,2}$.

Here

$q_{L}^{2}=\frac{\omega^{2}}{V_{L}^{2}}=\frac{\rho \omega^{2}}{\lambda+2 \mu} ; \quad q_{T}^{2}=\frac{\omega^{2}}{V_{T}^{2}}=\frac{\rho \omega^{2}}{\mu}$

Let in cylindrical coordinates $r, q, z$

$\phi_{j}(r, \vartheta, z, t)=f_{j}(r) e^{i q z+i n \vartheta-i \omega t} \quad(j=0,1,2)$.

Substituting expression (5) in (3), one obtains the following equation in $f_{i}$ : 


\section{A.A. Demidenko, V.A. Kochelap: Generation of coherent confined acoustic ...}

$\left(\frac{\partial^{2}}{\partial r^{2}}+\frac{1}{r} \frac{\partial}{\partial r}-k_{j}^{2}-\frac{n^{2}}{r^{2}}\right) f_{j}(r)=0$

where

$$
k_{0}^{2} \equiv k_{L}^{2}=q^{2}-q_{L}^{2} ; \quad k_{1,2}^{2} \equiv k_{T}^{2}=q^{2}-q_{T}^{2}
$$

In cylindrical coordinates the components of displacement vector $\vec{U}$ are, according to (2):

$$
\begin{aligned}
& U_{r}=\frac{\partial \phi_{0}}{\partial r}+\frac{1}{r} \frac{\partial \phi_{1}}{\partial \vartheta}+\frac{\partial^{2} \phi_{2}}{\partial r \partial z} ; \\
& U_{\vartheta}=\frac{1}{r} \frac{\partial \phi_{0}}{\partial \vartheta}-\frac{\partial \phi_{1}}{\partial r}+\frac{1}{r} \frac{\partial^{2} \phi_{2}}{\partial \vartheta \partial z} ; \\
& U_{z}=\frac{\partial \phi_{0}}{\partial z}-\frac{\partial^{2} \phi_{2}}{\partial r^{2}}-\frac{1}{r} \frac{\partial \phi_{2}}{\partial r}-\frac{1}{r^{2}} \frac{\partial^{2} \phi_{2}}{\partial \vartheta^{2}}
\end{aligned}
$$

Following are the stress tensor components that will be required later [5]:

$$
\begin{aligned}
\sigma_{r r} & =(\lambda+2 \mu) \operatorname{div} \vec{U}-2 \mu\left(\frac{1}{r} U_{r}+\frac{1}{r} \frac{\partial U_{\vartheta}}{\partial \vartheta}+\frac{\partial U_{z}}{\partial z}\right) ; \\
\sigma_{r \vartheta} & =\mu\left(\frac{1}{r} \frac{\partial U_{r}}{\partial \vartheta}+\frac{\partial U_{\vartheta}}{\partial r}-\frac{U_{\vartheta}}{r}\right) ; \\
\sigma_{r z} & =\mu\left(\frac{\partial U_{r}}{\partial z}+\frac{\partial U_{z}}{\partial r}\right) .
\end{aligned}
$$

Similar equations should be written down for the outer medium too (let us remind that all the quantities related to this medium are designated by an overscribed bar).

3. Further solution procedure is as follows. One should solve equations (6) both inside and outside of the medium. The solutions obtained involve twelve constants. Six of them are to be determined from the conditions at $r \rightarrow 0$ and $r \rightarrow \infty$. The rest of the constants are to be determined from the conditions that the displacements (8) and stress tensor components (9) are equal inside and outside of the cylinder at $r=R$. In this case the signs of $k_{j}^{2}, \bar{k}_{j}^{2}$ (7) are of essence. At various combinations, depending on the wave vector $q$ and media parameter values, one can obtain localized waves, surface waves (interface modes) that die off on both sides of the cylinder lateral surface, etc. Many such cases have been considered in $[6,7]$.

In particular, at $k_{L}^{2}<0 \quad \bar{k}_{L}^{2}>0$ localized waves arise, and the situation resembles the case of a free cylinder when vacuum serves as an outer medium. One can get the dispersion equations by equating the six-order determinant of the set of equations for boundary conditions to zero. For every integer $n \geq 0$ in expression (6) the dispersion equations define a class of normal waves. The value $n=1$ corresponds to a usual flexural wave similar to that in a plate. The case $n \geq 2$ defines a class of flexural waves of circular order $n$. No such waves exist in a plate.
When $n=0$, then dependence on $\theta$ disappears and the set of equations may be separated. Those in $U_{\theta}$ and $\sigma_{r \theta}$ (see (8), (9)) determine a class of torsional waves (the elementary discs rotate as a whole). The rest of equations determine a class of longitudinal (dilatometric) normal waves. Even in this special case the dispersion equation (being similar to Pochhammer equation [5] for a free cylinder) is very complicated and admits numerical solution only $[6,7]$.

For $n=0$ the boundary conditions for the longitudinal waves at the lateral surface of a cylinder $(r=R)$,

1) $U_{r}=\bar{U}_{r}$;2) $U_{z}=\bar{U}_{z}$;3) $\sigma_{r r}=\bar{\sigma}_{r r}$;4) $\sigma_{r z}=\bar{\sigma}_{r z}$,

are of the following form (with allowance made for dependence on $z(5))$ :

1) $\frac{\partial f_{0}}{\partial r}+i q \frac{\partial f_{2}}{\partial r}=\frac{\partial \bar{f}_{0}}{\partial r}+i q \frac{\partial \bar{f}_{2}}{\partial r}$;

2) $i q f_{0}-k_{T}^{2} f_{2}=i q \bar{f}_{0}-\bar{k}_{T}^{2} \bar{f}_{2}$;

3) $(\lambda+2 \mu) \operatorname{div} \vec{U}-2 \mu\left(\frac{U_{r}}{r}+i q U_{z}\right)=$;

$$
=(\bar{\lambda}+2 \bar{\mu}) \operatorname{div} \overline{\vec{U}}-2 \bar{\mu}\left(\frac{\bar{U}_{r}}{r}+i q \bar{U}_{z}\right)
$$

4) $\mu\left(i q U_{r}+\frac{\partial U_{z}}{\partial r}\right)=\mu\left(i q \bar{U}_{r}+\frac{\partial \bar{U}_{z}}{\partial r}\right)$.

4. The main objective of this work is to investigate the interaction via deformation potential between a localized acoustic wave and electrons drifting inside a cylinder. Taking into account expressions (2) and (3), one may write down the interaction Hamiltonian as

$H_{\text {int }}=b \operatorname{div} \vec{U}=-b q_{L}^{2} \phi_{0}$

here $b$ is the deformation potential constant. One can immediately see from (2) that $\operatorname{div} \vec{U}$ is determined by the potential $f_{0}$ only. The amplitude of $f_{0}$, however, is to be determined from the set of equations (10) and is related to the amplitudes $\bar{\phi}_{0}, \phi_{2}$ and $\bar{\phi}_{2}$. As a result, the problem becomes very complicated, as was stated above. To circumvent this, we shall use the model that we have applied previously to calculations for a plate $[1,3]$. Let us assume that the only difference (in a mechanical sense) between the two media, $\bar{A}$ and $A$, is that $\bar{\lambda} \neq \lambda$, while $\bar{\mu}=\mu$ and $\bar{\rho}=\rho$. Such a model retains all the main features of the problem studied, but allows, at the same time, to perform mathematical manipulation to the very end and obtain results in the analytical form.

Let us designate

$$
\frac{\bar{\lambda}+2 \mu}{\lambda+2 \mu}=h
$$




\section{A.A. Demidenko, V.A. Kochelap: Generation of coherent confined acoustic ...}

According to expressions (4) and (7), in the context of our model

$\bar{q}_{T}^{2}=q_{T}^{2} ; \quad \bar{k}_{T}^{2}=k_{T}^{2} ; \quad \bar{q}_{L}^{2}=\frac{1}{h} q_{L}^{2}$

It is easy to verify that the set of equations for boundary conditions (10) now reduces to the following two independent pairs of equations:

$f_{0}=\bar{f}_{0}$

$\frac{\partial f_{0}}{\partial r}=\frac{\partial \bar{f}_{0}}{\partial r} \quad r=R$

$f_{2}=\bar{f}_{2}$

$\frac{\partial f_{2}}{\partial r}=\frac{\partial \bar{f}_{2}}{\partial r}$

$$
r=R
$$

Equation (6) in $f_{0}$ at $n=0$ is the Bessel equation of zero order. We seek for localized solutions that go down with $r$ outside of the cylinder; so it should be $\bar{k}_{L}^{2}<0$ (7), i.e., $q<\bar{q}_{L}$. In order that solutions exist, it is necessary (as will be shown later) that $k_{L}^{2}>0$, i.e., $q>q_{L}$. From this it follows, according to (13), that $q_{L}>\bar{q}_{L}$, i.e., $h>1$. Therefore, according to (12), in order that localized solutions exist, the inner medium is to be «more soft», as in the case of a plate [1].

Let us denote

$\gamma^{2}=-k_{L}^{2}$

The solutions of Eq. (6) that are finite at zero are of the form

$$
f_{0}(r)=A_{0} J_{0}(\gamma r) \quad r<R
$$

where $J_{0}$ is the zero-order Bessel function of the first kind.

Outside of the cylinder $(r>R)$ the solution that dies out when $r \rightarrow \infty$ is

$$
\bar{f}_{0}(r)=\bar{A}_{0} K_{0}\left(\bar{k}_{L} r\right) \quad r>R
$$

where $K_{0}$ is the modified zero-order Bessel function of the second kind [8].

The dispersion equation and ratio between constants are determined from the set of equations for boundary conditions (14a):

$$
\begin{aligned}
& \bar{A}_{0}=A_{0} \frac{J_{0}(\xi)}{K_{0}(\eta)}=A_{0} \frac{\xi}{\eta} \frac{J_{1}(\xi)}{K_{1}(\eta)} \\
& \eta \frac{K_{1}(\eta)}{K_{0}(\eta)}=\xi \frac{J_{1}(\xi)}{J_{0}(\xi)}
\end{aligned}
$$

Here the dimensionless variables

$$
\begin{gathered}
\xi=\gamma R=R \sqrt{q_{L}^{2}-q^{2}} ; \\
\eta=\bar{k}_{L} R=R \sqrt{q^{2}-\bar{q}_{L}^{2}}
\end{gathered}
$$

are introduced.

According to the properties of Bessel functions, $J_{1}(i \xi)=$ $=i I_{1}(\xi), J_{0}(i \xi)=I_{0}(\xi)$ (here $I_{0}$ and $I_{1}$ are the modified Bessel functions of the first kind [8]). One can see from the dispersion equation that if it were $k_{L}^{2}>0$, i.e. $\gamma^{2}<0$ (15) (this corresponds to replacement $\xi \rightarrow i \xi$ in (19)), then the righthand side of equation (19) becomes negative. This means that the dispersion equation has no solutions in this case, since all the functions entering it are positive.

The dispersion equation (19) determines an infinite class of dispersion branches. At $x<<1$ the zero branch is of the following form [8]:

$\eta=\exp \left(-1 / \xi^{2}\right)$.

One can see that $h(20)$ is exponentially small; therefore $\bar{q}_{L}=q$ or $\omega=\bar{V}_{L} q$. Let us denote the consecutive zeros of the functions $J_{0}(\xi)$ and $J_{1}(\xi)$ by $\xi_{0, n}$ and $\xi_{1, n}$, respectively: $\xi_{0,1}=2.4 ; \xi_{0,2}=5.52 ; \xi_{0,3}=8.65 ; \xi_{1,1}=3.83 ; \xi_{1,2}=$ $=7.01 ; x_{1,3}=10.7$. The $\xi_{1, n}$ value determines a point where the $n$-th branch appears. Since $J_{1}=-J_{0}^{\prime}$, zeros of numerator in the right-hand side of equation (19) correspond to extrema of denominator. Immediately after the appearance point $J_{1}$ and $J_{0}$ are of the same sign, i.e., the right-hand side of equation (19) is positive as it must, since the left-hand side is always positive. Thus allowed $\xi$ values lie in the interval $\xi_{1, n}<\xi<\xi_{0, n+1}$. When $\xi$ passes through these intervals, then the frequency and wave vector change from their values at the appearance points

$\omega=\omega_{v, 0}=\xi_{1, v} \bar{V}_{L} / R \sqrt{h-1} ;$

$q=q_{v}^{0}=\xi_{1, v} / R \sqrt{h-1}$

to infinity. All the branches lie on the plot between the rays $\omega=V_{L} q$ and $\omega=\bar{V}_{L} q$. In a qualitative sense the situation is similar to that with a plate [1].

At sound frequencies approaching the $\mathrm{THz}$ band (that will be of predominant interest for us) the sound wavelength is of the order of Fermi wavelength of electron. Therefore, when dealing with electron-phonon interaction, one should not treat sound as a classical wave; it should be considered as a flux of quantized phonons. To perform quantization, let us introduce a length $L$ along $z$ (the direction of wave propagation) and impose the cyclic boundary conditions. Then

$q=\frac{2 \pi}{L} n(n=1,2, \ldots)$

The constant $A_{0}$ is to be determined from the normalization conditions. This is equivalent to setting the total wave energy equal to $\hbar \omega$. 


\section{A.A. Demidenko, V.A. Kochelap: Generation of coherent confined acoustic ...}

Generally the expressions for the strain tensor components in cylindrical coordinates are as follows [9]:

$$
\begin{aligned}
& S_{r r}=\frac{\partial U_{r}}{\partial r} ; S_{\vartheta \vartheta}=\frac{1}{r} \frac{\partial U_{\vartheta}}{\partial \vartheta}+\frac{U_{r}}{r} ; S_{z z}=\frac{\partial U_{z}}{\partial z} ; \\
& 2 S_{r z}=\frac{\partial U_{r}}{\partial z}+\frac{\partial U_{z}}{\partial r} ; 2 S_{r \vartheta}=\frac{\partial U_{\vartheta}}{\partial r}-\frac{U_{\vartheta}}{r}+\frac{1}{r} \frac{\partial U_{r}}{\partial \vartheta} ; \\
& 2 S_{\vartheta z}=\frac{1}{r} \frac{\partial U_{z}}{\partial \vartheta}+\frac{\partial U_{\vartheta}}{\partial z}
\end{aligned}
$$

For the wave under consideration $U_{q}=0, \frac{\partial}{\partial \vartheta}=0$. The potential energy is determined as

$$
\begin{aligned}
& U=\int_{V}\left\{\frac{1}{2}(\lambda+2 \mu)\left(S_{r r}+S_{\vartheta \vartheta}+S_{z z}\right)+\right. \\
& \left.+2 \mu\left(S_{r r} S_{\vartheta \vartheta}+S_{r r} S_{z z}+S_{\vartheta \vartheta} S_{z z}-S_{r z}^{2}\right)\right\} d V
\end{aligned}
$$

Here the integration is performed over the whole volume. It is not our intention to dwell in detail on $U$ calculation: being rather cumbersome, it does not involve any fundamental difficulties. (For all the necessary integrals involving Bessel functions one should refer, e.g., to [10].) The following two items, however, are to be noted. The term in the second parentheses in expression (24) that is multiplied by $m$ may be omitted because contributions from the cylinder and the rest of the volume cancel out. This stems from our model for which $\bar{\mu}=\mu, \bar{\rho}=\rho$. The kinetic energy turns out to be equal to the potential one as it must due to Clausius virial theorem. Therefore the total energy is twice the potential energy, and the constant $A_{0}$ may be determined from the following expression:

$$
\begin{aligned}
& 2 U=\frac{\pi}{2} L R^{2} \frac{\rho^{2} \omega^{4}(h-1)}{\bar{\lambda}+2 \mu} \times \\
& \times A_{0}^{2}\left[J_{0}^{2}(\xi)+\frac{\left(q R^{2}\right)}{\eta^{2}} J_{1}^{2}(\xi)\right]=\hbar \omega
\end{aligned}
$$

5. To this point we have not restricted the transverse size of the cylinder in any way. Going to construction of electronic states inside the cylinder, we shall assume that its radius $R$ is of the order of, or less than, the Fermi wavelength of electron. This means that we shall consider a quantum wire. In this case the transverse motion is quantized, while the longitudinal one may be treated using the quasiclassical approach. The electron potential energy will be considered to be zero inside a wire with infinite walls at its lateral surface. Let us seek for solution to Schrцdinger equation

$\Delta \psi+\frac{2 m}{\hbar^{2}} E \psi=0$

in the following form:
$\psi=\frac{1}{\sqrt{L}} \chi(r) e^{i k z+i n \vartheta}$

After substitution of (27) to equation (26) one obtains the following equation in $\chi(r)$ :

$\chi^{\prime \prime}+\frac{1}{r} \chi^{\prime}+\left(\lambda^{2}-\frac{n^{2}}{r^{2}}\right) \chi=0$

Here $\lambda$ is the eigenvalue parameter:

$\lambda^{2}=\frac{2 m}{\hbar^{2}} E-k^{2}$

The solution of (28) that remains finite at $r=0$ is:

$\chi(r)=C J_{n}(\lambda r)$

here $J_{n}$ is the $n$-th order Bessel function of the first kind. The boundary conditions (that determine the quantization conditions) for the wave function at $r=R$ are of the following form:

$J_{n}(l R)=0$.

Let $x_{n j}$ be the $j$-th root of equation $J_{n}(x)=0$. Then the energy that depends on three quantum numbers, namely, $k$ (continuous) and $n, j$ (discrete) is:

$E_{n j}(k)=\epsilon_{n j}+\frac{\hbar^{2} k^{2}}{2 m} ; \quad \epsilon_{n j}=\frac{\hbar^{2} x_{n j}^{2}}{2 m R^{2}}$

The lowest level (bottom of the lowest subband) corresponds to the root $x_{01}=2.4(n=1, j=1)$; next level corresponds to the root $x_{11}=3.83(n=0, j=1)$. The normalized wave function of the lowest subband is:

$\Psi_{0,1, k}=\frac{1}{\sqrt{L}} \chi_{01}(r) e^{i k z}$

Here the radial function is:

$\chi_{01}(r)=\frac{1}{J_{1}\left(x_{01}\right) \sqrt{\pi R^{2}}} J_{0}\left(x_{01} \frac{r}{R}\right)$

When calculating interaction between electrons and phonons, we shall consider the lowest subband to be filled and the next one empty. This means that the difference $\epsilon_{11}-\epsilon_{01}$ is to be over the Fermi energy. Later we shall estimate what are the restrictions imposed on $R$ by this requirement. In addition, we shall assume the above difference to be big as compared to the phonon energy $\hbar \omega$. In this case interaction induces not intersubband transitions but only electron scattering within the lowest subband. 
Taking interaction as weak, we shall consider it in the first order of perturbation theory. A probability for an electron to pass per unit time from a state with wave vector $k$ to that with wave vector $k^{\prime}$ and emit $(+)$ or absorb (-) a localized phonon with wave vector $q$ and energy $\hbar \omega(q)=\hbar S q$ (here $S$ is the phonon phase velocity that generally depends on $q$ ) is given by the well-known expression (for volume waves see [11, 12], for a quantum well see [3]):

$$
\begin{aligned}
& P^{ \pm}\left(k, k^{\prime} / q\right)=\frac{2 \pi}{\hbar}\left|\left\langle k\left|H_{\text {int }}\right| k^{\prime}\right\rangle\right|^{2} \times \\
& \times\left(N_{q}+\frac{1}{2} \pm \frac{1}{2}\right) \delta\left[E(k)-E\left(k^{\prime}\right) \pm \hbar S q\right]
\end{aligned}
$$

Here $N_{q}$ are the occupation numbers for the phonon mode.

A matrix element of the interaction energy (11) taken on the wave functions (33), (34) is:

$$
\left\langle k\left|H_{\text {int }}\right| k^{\prime}\right\rangle=M_{01} \delta_{k, k^{\prime} \pm q}
$$

the $\delta$-function in (36) (arising at integration over $z$ ) ensures conservation of wave vector.

Calculation of the matrix element (37) using (11) and (34) gives:

$$
M_{01}=b A_{0} q_{L}^{2} \Phi(\xi)
$$

Here a dimensionless function

$$
\Phi(\xi)=\frac{2}{J_{1}^{2}\left(x_{01}\right)} \int_{0}^{1} J_{0}(\xi t) J_{0}^{2}\left(x_{01} t\right) t d t
$$

is introduced. At small $x$ this function is close to unity up to $x \sim 2$.

The total probability of one-phonon emission (absorption) is obtained by summing expression (35) over all the initial and final electronic states:

$$
P^{ \pm}(q)=\sum_{k, k^{\prime}} P^{ \pm}\left(k, k^{\prime} / q\right) F(k)\left[1-F\left(k^{\prime}\right)\right]
$$

here $F(k)$ is the 1D electron distribution function [13]. It is not our intention to dwell in detail on the standard procedure of calculating the sum (39). For the volume case and a quantum well it has been done in $[11,12]$ and [3], respectively.

Let us consider a case when a constant electric field $E_{0}$ is applied along the wire. We shall assume it to be not too high, so that one may neglect the electron gas heating. The Fermi-Dirac distribution function shifted by the drift velocity $V_{d}=\mu E_{0}$ ( $m$ is mobility) will serve as a $1 \mathrm{D}$ electron distribution function. Let us consider the phonon mode to be sufficiently excited, so that $N_{q}$ is much greater than unity (i.e., one may neglect spontaneous phonon emission). In this case the pure probability of coherent phonons emission (generation) per unit time is:

$$
\begin{aligned}
& P^{+}-P^{-}=\frac{2 \pi}{\hbar}\left|M_{01}\right|^{2} N_{q} \frac{m L}{\hbar^{2} q 2 \pi} \\
& {\left[F\left(\frac{1}{2} q+\frac{m\left(S-V_{d}\right)}{\hbar}\right)-F\left(-\frac{1}{2} q+\frac{m\left(S-V_{d}\right)}{\hbar}\right)\right]}
\end{aligned}
$$

It is easy to verify that the term in brackets in expression (40) may be positive (i.e., induced emission exceeds absorption) at any temperature for both degenerate and nondegenerate statistics if the Cerenkov criterion is fulfilled:

$V_{d}>S$

For the Fermi-Dirac statistics at absolute zero the above term in brackets is non-zero when

$$
2 k_{F}-2 \frac{m}{\hbar}\left|V_{d}-S\right|<q<2 k_{F}+2 \frac{m}{\hbar}\left|V_{d}-S\right|
$$

It is 1 when inequality (41) is obeyed and -1 if the inequality (41) fails. It should be noted that in the case of a quantum well [3] there was no restriction on $q$ from below. If the left inequality (42) fails, then both emission and absorption are non-zero and compensate each other. If the right inequality (42) fails, then both emission and absorption are absent. The above regularities may be described using two Heaviside unit functions (see below).

The absorption coefficient per unit length is obtained through dividing $P^{-}-P^{+}$by the phonon flux $N_{q} V_{S}$ where $V_{S}$ is the phonon group velocity. As in the case of a quantum well [1], all the dispersion branches $\omega(q)$ lie inside the wedge made by the rays $\omega=V_{L} q$ and $\omega=\bar{V}_{L} q$. At $h-1<<1$ this wedge is narrow, so the group velocity $V_{S}$ is not too different from the phase velocity $S$, and the latter depends on $q$ but slightly. Replacing in a flux $V_{S}$ by $S$, one obtains for the $j$-th dispersion branch the following expression:

$$
\begin{aligned}
& \alpha_{0 j}=-\frac{2 m b^{2}}{\hbar^{2} \pi R^{2}(\lambda+2 \mu)}\left(\frac{h}{h-1}\right) \frac{\Phi^{2}\left(\xi_{j}\right)}{J_{0}^{2}\left(\xi_{j}\right)+\frac{\left(q R^{2}\right)}{\eta^{2}} J_{1}^{2}\left(\xi_{j}\right)} \times \\
& \times\left[\Theta\left(2 k_{F}-q+\frac{2 m\left(V_{d}-S\right)}{\hbar}\right)-\right.
\end{aligned}
$$$$
\left.-\Theta\left(2 k_{F}-q-\frac{2 m\left(V_{d}-S\right)}{\hbar}\right)\right]
$$

6. To make numerical estimates, let us use the parameters that correspond to GaAs crystal: $\lambda+2 \mu=1.18$. $\cdot 10^{12}$ dyne $/ \mathrm{cm}^{2} ; \rho=5.317 \mathrm{~g} / \mathrm{cm}^{3} ; V_{L}=4.7 \cdot 10^{5} \mathrm{~cm} / \mathrm{s} ; m=$ $=0.067 m_{0} ; b=-8 \mathrm{eV}$. Let the wire radius $R$ and $h$ are $8 \mathrm{~nm}$ and 1.1, respectively. Take the equilibrium linear electron concentration in a wire $N_{l}=2 \cdot 10^{6} \mathrm{~cm}^{-1}$; this corresponds to the bulk concentration $N=10^{18} \mathrm{~cm}^{-3}$. In this case [13] $k_{F}=\pi N_{l} / 2 \cong 3 \cdot 10^{6} \mathrm{~cm}^{-1}$ and the Fermi energy $E_{F}=57 \mathrm{meV}$. At the chosen $R$ value the first and second 


\section{A.A. Demidenko, V.A. Kochelap: Generation of coherent confined acoustic ...}

energy levels for transverse motion are $\varepsilon_{1}=51 \mathrm{meV}$ and $\varepsilon_{2}=131 \mathrm{meV}$, respectively. Thus $\varepsilon_{2}-\varepsilon_{1}>E_{F}$, as was assumed above. Let the constant electric field $E_{0}=20 \mathrm{~V} / \mathrm{cm}$; then, at mobility $\mu=10^{5} \mathrm{~cm}^{2} / \mathrm{V} \cdot \mathrm{s}$, we get $V_{d}=2 \cdot 10^{6} \mathrm{~cm} / \mathrm{s}>$ $>S \approx V_{L}$.

Before proceeding to numerical estimations, let us perform a general analysis of the expression (43) for $\alpha$.

1) At the points where non-zero branches $\xi_{0 j}$ arise $J_{1}\left(\xi_{0 j}\right) \rightarrow 0$, and, according to the dispersion equation (19), $\eta \rightarrow 0$. In this case, however [8],

$$
\frac{J_{1}^{2}\left(\xi_{0 j}\right)}{\eta^{2}}=\frac{J_{0}^{2}\left(\xi_{0 j}\right)}{\xi_{0 j}^{2}} \cdot \frac{K_{1}^{2}(\eta)}{K_{0}^{2}(\eta)} \propto \frac{1}{(\eta \ln \eta)^{2}} \rightarrow \infty
$$

i.e., according to (43), $\alpha=0$. When moving off the above points, the ratio, say, $\alpha_{01} / \alpha_{00}$, approaches 1 as $q$ grows.

2) Let us consider the limiting case of a spatially uniform medium (this corresponds to the limit $h \rightarrow 1$ ). If, more particularly, $\xi^{2}=(h-1)(q R)^{2}<<1$, then $h$ is exponentially small, in accordance with (21). The index of wave spatial damping, $\bar{k}=\eta / R$, as well as the ratio between the inner and external energies, $U_{i} / U_{e}$, approach zero. This means that the wave becomes the volume one. In this case the denominator in expression (43) is exponentially big and $\alpha \rightarrow 0$, i.e., a volume wave cannot be excited and amplified by electron drift in a narrow quantum wire. At the above medium parameters and wire radius $R=8 \mathrm{~nm}$ let us take $q=6 \cdot 10^{6} \mathrm{~cm}^{-1} \approx 2 k_{F}$; this corresponds to the frequency of $450 \mathrm{GHz}\left(\omega=2.8 \cdot 10^{12} \mathrm{~s}^{-1}\right)$. In this case, according to expression (43), $\alpha_{00}=-3 \cdot 10^{3} \mathrm{~cm}^{-1}$. This $\alpha$ value is close to that $\left(-1.3 \cdot 10^{3} \mathrm{~cm}^{-1}\right)$ for a quantum well $10 \mathrm{~nm}$ thick at the same frequency [3].

\section{Acknowledgements}

We are grateful to Dr. V.I. Sheka for assistance in calculations and E.V. Mozdor for her help in preparation of the manuscript. The work was supported by the State Fundamental Researches Foundation of Ukraine (Grant № 2.4/679) and by the project STCU № Gr-01.

\section{References}

1. A.A. Demidenko, V.A. Kochelap, Amplification of localized acoustic waves by the electron drift in a quantum well // Semiconductor Physics, Quantum Electronics \& Optoelectronics 2(1), pp.11-24 (1999).

2. A.A. Demidenko, V.A. Kochelap, E.F. Venger, Piezoelectrically active acoustic waves confined in a quantum well and their amplification by electron drift // Semiconductor Physics, Quantum Electronics \& Optoelectronics, 3(4), pp. 427-431 (2000).

3. S.M. Komirenko, K.W. Kim, A.A. Demidenko, V.A. Kochelap, M.A. Stroscio, Generation and aplication of sub-THz coherent acoustic phonons under the driff of two-dimensional electrons // Phys.Rew.B, 62(11), pp. 7459-7469.

4. L.D. Landau, E.M. Lifshits, Theory of Elasticity (in Russian), Nauka, Moscow (1987).

5. T. Micker, A. Meitzler, Guided-wave propagation in extended cylinders and plates (Russian translation from Physical Acoustics. Principles and Methods, Ed. W.P. Mason, 1, Pt. A, Academic Press, New York and London).

6. N. Nishiguchi, Confined and interface acoustic phonons in a quantum wire // Phys. Rev. B, 50(15), p.10970 (1994).

7. N. Nishiguchi, Resonant acoustic-phonon modes in a quantum wire // Phys. Rev. B, 52, p.5279 (1995).

8. A Handbook of Special Functions (in Russian), Nauka, Moscow (1979).

9. A.E. Love, A Treatise on the Mathematical Theory of Elasticity, London and New York (1927).

10. A.P. Prudnikov, Yu.A. Bychkov, O.I. Marichev, Integrals and Series. Special Functions (in Russian), Nauka, Moscow (1983).

11. J. McFee, Propagation and amplification of sonic waves in piezoelectric semiconductors (Russian translation from Physical Acoustics. Principles and Methods, Ed. W.P. Mason, 4, Pt. A, Academic Press, New York and London).

12. J.W. Tucker, V.W. Rampton, Microwave Ultrasonics in Solid State Physics, North Holland Publ. Co., Amsterdam (1972).

13. V.V. Mitin, V.A. Kochelap, M.A. Stroscio, Quantum Heterostructures for Microelectronics and Optoelectronics, Cambridge University Press, New-York-Cambridge (1998). 